\title{
Syndecans and CD44 in Normal Human Keratinocyte Cultures: Modulation with Medium Composition and All-trans Retinoic Acid
}

\author{
M-A. Le Bitoux ${ }^{\mathrm{a}}$, S. Callejon ${ }^{\mathrm{a}}$, M. Rodriguez Aller ${ }^{\mathrm{a}}$, A. Denis ${ }^{\mathrm{b}}$, I. Pernet $^{\mathrm{b}}$ and M. Haftek $^{*}$,a \\ ${ }^{a}$ Université de Lyon, EA4169, "Normal and Pathological Functions of the Skin Barrier»; and ${ }^{b}$ Bioderma Laboratory, \\ Lyon, France
}

\begin{abstract}
We investigated chosen epidermal proteoglycans in different culture models to explore their expression by keratinocytes at various states of differentiation. Fully defined conditions of our epidermal reconstructions allowed for evaluation of the proteoglycans in "normal" or "ageing"-like epidermal tissues in vitro. The "ageing"-like epidermis was characterized by the viable layers' atrophy, low proliferation rate and premature keratinisation. Expression of all studied proteoglycan genes could be detected with quantitative real-time PCR but the syndecan-4 protein wasn't observed in the reconstructed epidermis using immunohistochemistry. Treatment with all-trans retinoic acid resulted in epithelial acanthosis, combined with up-regulation of proteoglycans in the "ageing"-like culture conditions. In "normal" reconstructed epidermis, retinoids increased the syndecan- 4 gene expression but down-regulated that of syndecan- 1 . The observed complex changes are compatible with the presumed but still poorly understood roles of various proteoglycans in epidermal differentiation. Our defined culture models are suitable for screening of pharmacological agents modulating epidermal proteoglycan expression.
\end{abstract}

Keywords: Reconstructed human epidermis, proteoglycans, all-trans retinoic acid, keratinocyte cultures.

\section{INTRODUCTION}

Proteoglycans (PGs) are complex glycoproteins associated with the extracellular matrix and cell surfaces. They are composed of a core protein to which one or several chains of glycosaminoglycans are covalently linked. These glycosaminoglycans belong to four families defined as heparan sulphate, chondroitin sulphate, keratan sulphate, and dermatan sulphate depending on their sugar composition, sulphatation and epimerization [1]. PGs play an important role in cell biology regulating cell proliferation, migration or differentiation [1-3]. In fact, they participate in various biological processes such as assembly of the extracellular matrix, attachment and storage of growth factors, cation fixation and ion filtration through the molecular network, tissue hydration, and cell attachment and adhesion [4].

All cells express at least one of the heparan sulphate proteoglycans on their surface, and all adherent cells, like keratinocytes, express one or several syndecans [5]. The syndecan family is formed from four known members. These heparan sulphate PGs are composed of a short C-terminal cytoplasmic domain, a highly conserved hydrophobic transmembrane domain and an N-terminal ectodomain. This extracellular part of the protein displays a specific sequence for each member and contains 3 to 5 sites necessary for glycosaminoglycan attachment [6]. Sometimes, syndecans may additionally bear chondroitin sulphate chains at these sites [7]. The ectodomains equipped with their long glycosaminoglycan chains confer to syndecans distinct binding

*Address correspondence to this author at the Université de Lyon, Laboratoire de Dermatologie, Pavillon R, Hôpital E Herriot, 69437 Lyon, France; Tel: +33 472110292; Fax: +33 472110290; E-mail: haftek@univ-lyon1.fr specificities, with various soluble and insoluble ligands, and can facilitate the formation of signaling complexes [8]. Moreover, the extracellular domains of the syndecans can be cleaved by specific ectoenzymes in several physiological situations, in order to liberate soluble heparan sulphate PG effectors [8-10]. For example, the shedding of syndecan-1 and -4 ectodomains in wounded tissues is a mechanism that permits sequestration of growth factors and maintenance of the proteolytic balance at the wound site [11, 12].

CD44, principally carrying chondroitin sulphate chains, is ubiquitously expressed on the surface of various cell types and is the major cellular receptor for hyaluronic acid [13]. The human CD44 gene is composed of at least 20 exons. Ten of them are constitutively expressed and the ten others can be alternatively spliced to produce many functionally distinct isoforms containing combinations of variant exons. The major isoform of CD44 expressed by keratinocytes is called epican (v3-v10 variant) [14], but some other variants of this PG can also be observed in the epidermis depending on the degree of cell differentiation [15]. Epican is a transmembrane chondroitin sulphate PG modified by the attachment of heparan sulphate chains [16-19]. In the human epidermis, CD44 co-localizes with hyaluronic acid in the epithelial intercellular spaces of basal and spinous cell layers, with a positive gradient following the differentiation [20, 21]. Two major functions of CD44 in the epidermis could be the regulation of keratinocyte proliferation in response to extracellular stimuli, and the maintenance of local hyaluronic acid homeostasis [22].

In fact, today's knowledge about involvement of these various heparan sulphate PGs in the epidermal cell behavior is limited and weakly documented. Kim et al. have studied 
syndecan-1, -2, -3 and -4 mRNA expression in mouse skin sections, and found the presence of only syndecan- 1 and -4 mRNAs in the primary cultures of mouse keratinocytes on northern blots [9]. The expression patterns of the syndecan-1, 2 and -4 proteins were described in normal human epidermis and in chronic leg ulcers by Lundqvist and Schmidtchen [20]. In the healthy skin, syndecan-1 appeared to be distributed pericellularly in all living epidermal layers, with a stronger expression in the suprabasal region $[20,23]$. In a fresh skin lesion, a transient decrease of syndecan- 1 and -4 expression was observed in keratinocytes migrating into the wound, whereas this expression was increased in the keratinocytes proliferating at the wound margin [24, 25]. Inversely, a lower expression of syndecan-1 was observed at the edge of a chronic ulcer [20]. In syndecan-1 knock out mice, as well as in mice overexpressing this PG, the wound healing was delayed because of the defective keratinocyte migration [26, 27]. These data highlight the involvement of syndecan-1 in epithelial wound healing processes and demonstrate the importance of a balance in PG levels for an optimal tissue repair.

The only description of syndecan-2 expression in the human epidermis indicated the presence of a weak, homogeneous, cytoplasmic staining in the keratinocytes of all living layers [20]. In healing chronic ulcers, the protruding basal keratinocyte layers lacked syndecan-2 expression. A previous study by Gallo et al. has failed to demonstrate any syndecan-2 protein expression in the tissue sections of human epidermis grafted onto nude mice, whereas the same antibody detected it in Western blots [25].

mRNA expression of syndecan-4 was found abundant in mouse skin keratinocytes [9] and the corresponding protein could be repeatedly demonstrated histochemically in normal human and mouse epidermal keratinocytes. Syndecan-4 shows a pericellular localization in keratinocytes of the basal and spinous layers [20, 25].

The aim of this study was to observe the expression patterns of different proteoglycans in the reconstructed epidermal tissues grown under various fully defined culture conditions. Due to the wide diversity of existing PG proteins, additionally complicated by the varying kinds of glycosaminoglycan attachment and the degrees of their sulphatation or acetylation, we have chosen not to address the glycosylation aspects this time. Potential implication of PG in the process of terminal differentiation of keratinocytes and epidermal barrier function, which are the aspects of special interest to our laboratory, have influenced our choice of molecules to be studied. Indeed, we have concentrated on the family of syndecans, with its prominent differentiation -related member syndecan 1, and the hyaluronic acid receptor CD44. Modulation of the keratinocyte proliferation and differentiation was achieved by changing the medium composition, leading to the development of "ageing"-like and "normal"like well-growing in vitro models of human epidermis. Epidermal cultures produced in the presence of a medium containing reduced quantities of growth factors presented morphological traits characteristic of the aged human epidermis, i.e. thin living compartment with fewer mitoses in the basal layer, premature keratinization and delayed, incomplete desquamation. Although these criteria are by no means specific for the "epidermal ageing" they represent morphological features observed during the ageing process in vivo [28] and, therefore, we decided to use them for evaluation of the in vitro reproduced condition. All-trans retinoic acid (ATRA) was used as a reference molecule to demonstrate the feasibility of the further prospective studies on test compounds. These future experiments shall be aimed at the analysis of the influence of drugs modulating PG expression on wound healing and epidermal ageing.

\section{MATERIALS AND METHODS}

\section{Keratinocyte Cultures and Production of Epidermal Equivalents}

Human keratinocytes were isolated from normal adult skin of patients undergoing abdominal plastic surgery, following patients' informed consent and according to institutional guidelines. Foreskins taken from children were also tested for comparison. Primary cultures of keratinocytes were established in Keratinocyte - Serum Free Medium supplemented with L-glutamine, epidermal growth factor (EGF) and bovine pituitary extract (KSFM; Invitrogen, Cergy Pontoise, France). Cultures were grown in a humidified $5 \% \mathrm{CO}_{2}$ atmosphere at $37^{\circ} \mathrm{C}$ and the medium was changed every 2-3 days. At $80 \%$ of confluence, keratinocytes were detached with $0.05 \%$ trypsin, $0.53 \mathrm{mM}$ EDTA (Invitrogen) and re-seeded on polycarbonate membrane inserts (Transwell $0.4 \mu \mathrm{m}$ pores; Corning, Amboise, France) at $5 \times 10^{5}$ cells per $\mathrm{cm}^{2}$, in a medium composed of a 3:1 mixture of Dulbecco's modified Eagle's medium (DMEM, high glucose) and Ham's F12 medium (both from Invitrogen) supplemented with : bovine serum albumin, 24 $\mu \mathrm{M}$; human EGF, $10 \mathrm{ng} / \mathrm{mL}$; insulin, $5 \mu \mathrm{g} / \mathrm{mL}$; hydrocortisone, $0.4 \mu \mathrm{g} / \mathrm{mL}$; isoproterenol, $1 \mu \mathrm{M}$; L-carnitin, $10 \mu \mathrm{M}$; Lserin, $10 \mathrm{mM}$; palmitic acid, $25 \mu \mathrm{M}$; linoleic acid, $15 \mu \mathrm{M}$; oleic acid, $25 \mu \mathrm{M}$; arachidonic acid, $7 \mu \mathrm{M}$; tocopherol acetate, $20 \mu \mathrm{M}$; Vitamin C, $40 \mu \mathrm{g} / \mathrm{mL}$ (all from Sigma-Aldrich; SaintQuentin Fallavier, France); gentamicin, $20 \mu \mathrm{g} / \mathrm{mL}$; $1 \%$ antibiotic/antimycotic solution (both from Invitrogen). This "normal" medium, composed according to Boyce and Williams [29] and Ponec et al. [30], was first removed 24h after seeding and then changed every 2-3 days. After 5 days in submerged conditions, keratinocytes were lifted at the air-liquid interface and cultured for 14 more days. During the emerged culture, the composition of the medium was modified in some cases in order to obtain premature "ageing"-like changes (medium with no vitamin C and EGF, with only $0.5 \mu \mathrm{g} / \mathrm{mL}$ of insulin). The resulting two kinds of cultures were referred to as "normal" and "ageing" -like.

Some of the immersed cultures were switched to the "normal" medium upon confluence and maintained submerged for 5 additional days to obtain stratified epidermal sheets.

\section{Treatment of "Normal" and "Ageing" -Like Recon- structed Epidermis with All-Trans Retinoic Acid (ATRA; R2625, Sigma-Aldrich)}

Six days after emersion of the cultures, when the epidermis showed complete differentiation, $10 \mathrm{nM}$ ATRA was added to the culture medium for twenty-four hours or eight more days. The control cultures, received only the solvent: 1 $\%$ DMSO (Sigma). Each experiment was repeated 8 times. Epidermal mRNAs were extracted 24 hours after the addition of ATRA, and the cultures used for histological analysis were stopped after 8 days of initiating the treatment. 
In a preliminary experiment, immersed keratinocyte cultures were treated with ATRA for 2, 4, 10, 24 and 48 hours to determine the optimal time for mRNA extraction, suitable for the PG gene expression studies (data not shown).

\section{Histology and Immunohistochemistry}

\section{Tissue Preparation}

The reconstructed epidermis samples and normal skin biopsies from the same donors were rinsed in phosphate buffered saline (PBS; Invitrogen) and fixed for 3 days at $4^{\circ} \mathrm{C}$ in ImmunoHistofix before being embedded in ImmunoHistowax (both from A Phase; Mormont, Belgium). Parts of each normal human skin biopsy were also snap-frozen in liquid nitrogen or fixed in Baker's formalin solution and embedded in paraffin using routine procedures. Immunohistochemical staining was performed on $4-\mu \mathrm{m}$ sections from all specimens. The dewaxed and rehydrated sections were stained in parallel with the native frozen tissue sections of the same skin donors, to establish the influence of the preparative techniques on the tissue reactivity with each and every antibody. Samples embedded in the classical paraffin were microwave pre-treated before staining in the antigen retrieval citrate buffer $(\mathrm{pH} 6$; Dako ChemMate, Trappes, France; 2 x 3min. at 700W).

Stratified epithelial sheets from confluent immersed cultures were detached from the plastic dishes with dispase (2.5 mg/mL, $15 \mathrm{~min}$. at $37^{\circ} \mathrm{C}$; Sigma-Aldrich), placed in a drop of Tissue-Tek medium (Miles Inc., Elkhart, USA) and snap-frozen in liquid nitrogen.

\section{Immunolabeling Procedures}

Standard hematoxylin-phloxine staining was used for routine evaluation of tissue morphology. The thickness of the viable part of the reconstructed epidermis was measured using ImageJ software (http://rsb.info.nih.gov/ij/). A monoclonal antibody to Ki67 antigen expressed in the nuclei of cycling cells (cone MIB-1, Dako Cytomation, Glostrup, Denmark) served for evaluation of keratinocyte proliferation. The quantitative results (at least 10 measurements per slide, performed on five cultures of each type) were statistically analyzed with Wilcoxon T-test.

Three monoclonal mouse IgG antibodies: anti-CD44v3 (clone VFF-327V3; dilution 1:30; Abcys SA, Paris, France), anti-CD44 H-CAM (clone DF1485, recognizing all forms of CD44; dilution 1:30; Santa Cruz Biotechnology, Santa Cruz, CA, USA), anti-syndecan-4 (clone 5G9; dilution 1:100; Santa Cruz Biotechnology), and two polyclonal rabbit antibodies, H174 anti-syndecan-1, M140 anti-syndecan-2 (both applied at 1:100 dilution; Santa Cruz Biotechnology) were used for localization of the respective PGs employing the LSAB2 kit (Dako, Carpinteria, CA, USA) and AEC (3amino-9-ethylcarbazole) peroxidase substrate (Invitrogen).

A biotinylated hyaluronic acid binding protein, b-HABP ( $5 \mu \mathrm{g} / \mathrm{mL}$; Seikagaku, Tokyo, Japan) was used with the same detection system to stain this free glycosaminoglycan.

Immunolabeled sections were counterstained with hematoxylin.

\section{Electron Microscopy}

For ultrastructural examination, small tissue fragments were fixed in $2 \%$ glutaraldehyde in sodium cacodylate buffer and post-fixed in $1 \%$ osmium tetroxide in the same buffer, before being dehydrated in graded ethanol series and embedded in Epon. Ultrathin sections were counterstained with uranyl acetate and lead citrate before observation in a transmission electron microscope (CM120, Philips, Eindhoven, Netherlands).

\section{Quantitative RT-PCR Evaluation of the Proteoglycans' mRNA Expression}

The level of mRNA expression of various proteoglycans was assessed with quantitative real time polymerase chain reaction (qRT-PCR). Emerged keratinocyte cultures, established in "normal" and "ageing" conditions were treated or not with ATRA. Total RNA was extracted using Trizol (Invitrogen). First strand cDNA synthesis was performed with $500 \mathrm{ng}$ of total RNA from each sample using avian

Table 1. Primers Used for Amplification of GAPDH and Proteoglycan mRNA -Derived cDNAs

\begin{tabular}{|c|c|c|c|c|c|c|}
\hline \multicolumn{2}{|c|}{ Target } & Sequence (5'- 3') & mRNA Product & Gene Product Size (bp) & Size (nt) & $\operatorname{Tm}\left({ }^{\circ} \mathbf{C}\right)$ \\
\hline \multirow{2}{*}{ GAPDH } & \multirow{2}{*}{ Sense Reverse } & 5'-AGCGAGATCCCTCCAAAATC-3' & \multirow{2}{*}{134} & \multirow{2}{*}{263} & \multirow{2}{*}{20} & \multirow{2}{*}{60} \\
\hline & & 5'-GGCAGAGATGATGACCCTTT-3' & & & & \\
\hline \multirow{2}{*}{ CD44 } & \multirow{2}{*}{$\begin{array}{c}\text { Sense } \\
\text { Reverse }\end{array}$} & 5'-CCCAGATGGAGAAAGCTCTG-3' & \multirow{2}{*}{146} & \multirow{2}{*}{3679} & \multirow{2}{*}{20} & \multirow{2}{*}{62} \\
\hline & & 5'-GGAGGTGTTGGATGTGAGGA-3' & & & & \\
\hline \multirow{2}{*}{ Syndecan-1 } & \multirow{2}{*}{$\begin{array}{c}\text { Sense } \\
\text { Reverse }\end{array}$} & 5'-CTTCACACTCCCCACACAGA-3' & \multirow{2}{*}{137} & \multirow{2}{*}{727} & \multirow{2}{*}{20} & \multirow{2}{*}{62} \\
\hline & & 5'-GTATTCTCCCCCGAGGTTTC-3' & & & & \\
\hline \multirow{2}{*}{ Syndecan-2 } & \multirow{2}{*}{$\begin{array}{c}\text { Sense } \\
\text { Reverse }\end{array}$} & 5'-CCAGCCGAAGAGGATACAAA-3' & \multirow{2}{*}{146} & \multirow{2}{*}{1060} & \multirow{2}{*}{20} & \multirow{2}{*}{60} \\
\hline & & 5'-ATGCGATACACCAACAGCAG-3' & & & & \\
\hline \multirow{2}{*}{ Syndecan-4 } & \multirow{2}{*}{$\begin{array}{c}\text { Sense } \\
\text { Reverse }\end{array}$} & 5'-TGAGGATGTGTCCAACAAGG-3' & \multirow{2}{*}{126} & \multirow{2}{*}{3076} & \multirow{2}{*}{20} & \multirow{2}{*}{60} \\
\hline & & 5'-AGGAAGACGGCAAAGAGGAT-3' & & & & \\
\hline
\end{tabular}

In order to prevent contamination of cDNA signal with that originating from the genomic DNA, all primer pairs' sequences were selected to cover an intron splicing site of each mRNA. The size of each amplicon was controlled on ethidium bromide-stained agarose gel and double-checked with the melting curves given by the LightCycler. bp : base pair; nt : nucleotide; Tm : melting temperature. 
myeloblastosis virus reverse transcriptase (Promega, Charbonnières, France), according to the manufacturer's instructions, in a Perkin - Elmer 9600 thermal cycler (Life and Analytical Sciences, Courtaboeuf, France). The complementary DNA equivalent of $8 \mathrm{ng}$ of total RNA was used for real time amplification using Fast Start SYBR Green I Master Mix (Roche, Mannheim, Germany), $2 \mathrm{mM} \mathrm{MgCl}_{2}$, and 0.5 $\mu \mathrm{M}$ concentrations of each primer (see selected primers in Table 1). The amplification process in the LightCycler 2.0 (Roche) was composed of an initial denaturation step at $95^{\circ} \mathrm{C}$ for $10 \mathrm{~min}$., followed by 45 cycles of denaturation $\left(95^{\circ} \mathrm{C}\right.$ for $10 \mathrm{sec}$.), hybridization $\left(59^{\circ} \mathrm{C}\right.$ for $\left.10 \mathrm{sec}.\right)$, and elongation $\left(72^{\circ} \mathrm{C}\right.$ for $6 \mathrm{sec}$.). The melting analysis for one cycle was as follows: up-ramp $\left(20^{\circ} \mathrm{C} / \mathrm{sec}\right.$. $)$ to melt at $95^{\circ} \mathrm{C}$ for $2 \mathrm{sec}$., down-ramp $\left(20^{\circ} \mathrm{C} / \mathrm{sec}\right.$.) to anneal at $65^{\circ} \mathrm{C}$ for 15 sec., and then slow up-ramp $\left(0.1^{\circ} \mathrm{C} / \mathrm{sec}\right.$. $)$ for continuous acquisition up to $95^{\circ} \mathrm{C}$. The specificity of each designed primer was ensured by Basic Local Alignment Search Tool analysis and visualization of the PCR products on ethidium bromide-stained agarose gels. As controls, we used total RNA extracted from normal human epidermis, previously separated from the dermis by enzymatic treatment with dispase $\left(2.5 \mathrm{mg} / \mathrm{mL}, 1\right.$ hour at $37^{\circ} \mathrm{C}$; Sigma-Aldrich). The reaction efficiency was determined with a cDNA dilution series. In the negative control cDNA was substituted with distilled water. Before quantification, normalization of mRNA sam- ples was performed using the GAPDH mRNA as a reference gene; its expression did not change in response to the ATRA treatments applied (data not shown). The relative level of each mRNA was calculated with the RelQuant software (Roche) as indicated in the user's manual. The results were expressed in arbitrary units as a ratio between the values found for a given gene in the ATRA-treated cultures and their control, non-treated counterparts (mean values of eight experiments \pm SD). The statistical significance was determined using the non-parametric Wilcoxon T-test.

\section{RESULTS}

\section{Tissue Morphology}

In Vitro Reconstructed Epidermis Presented Well Developed Keratinocyte Layers Closely Resembling Normal Human Tissue

Submerged keratinocyte cultures stratified in the "normal" medium to produce 4-5 layers -thick epidermal sheets (Fig. 1). There was no horny layer but occasionally individual cornified cells could be observed at the top of the cultures on the 5 th day after confluence.

Our emerged cultures showed normal -looking stratification and keratinization after emersion and maintenance with the fully supplemented "normal" medium (Fig. 2, first column). The obtained reconstructed epidermis normally ex-

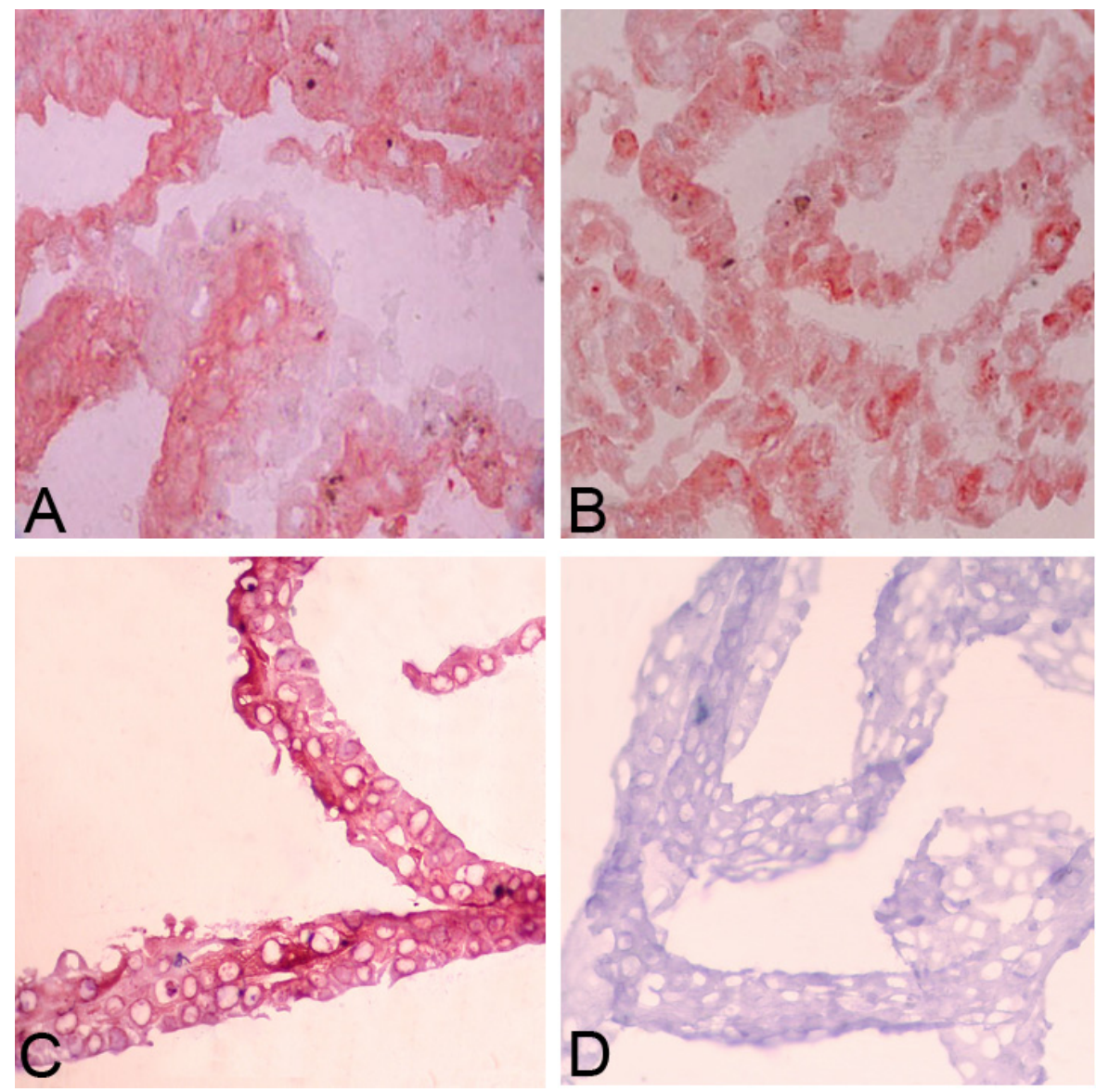

Fig. (1). PG labeling in submerged cultures. Submerged cultures of human keratinocytes in "normal", fully supplemented growing medium stratify and express CD44 H-CAM (A), Syndecan-1 (B) and Syndecan-2 (C), but not Syndecan-4 (D) (5 days after confluence; frozen tissue sections; red staining, blue counterstaining). Epidermal sheets were detached from plastic dishes with dispase wrapped in Tissue-Tek and snap-frozen in liquid nitrogen. All pictures were taken at x 400 magnification. 


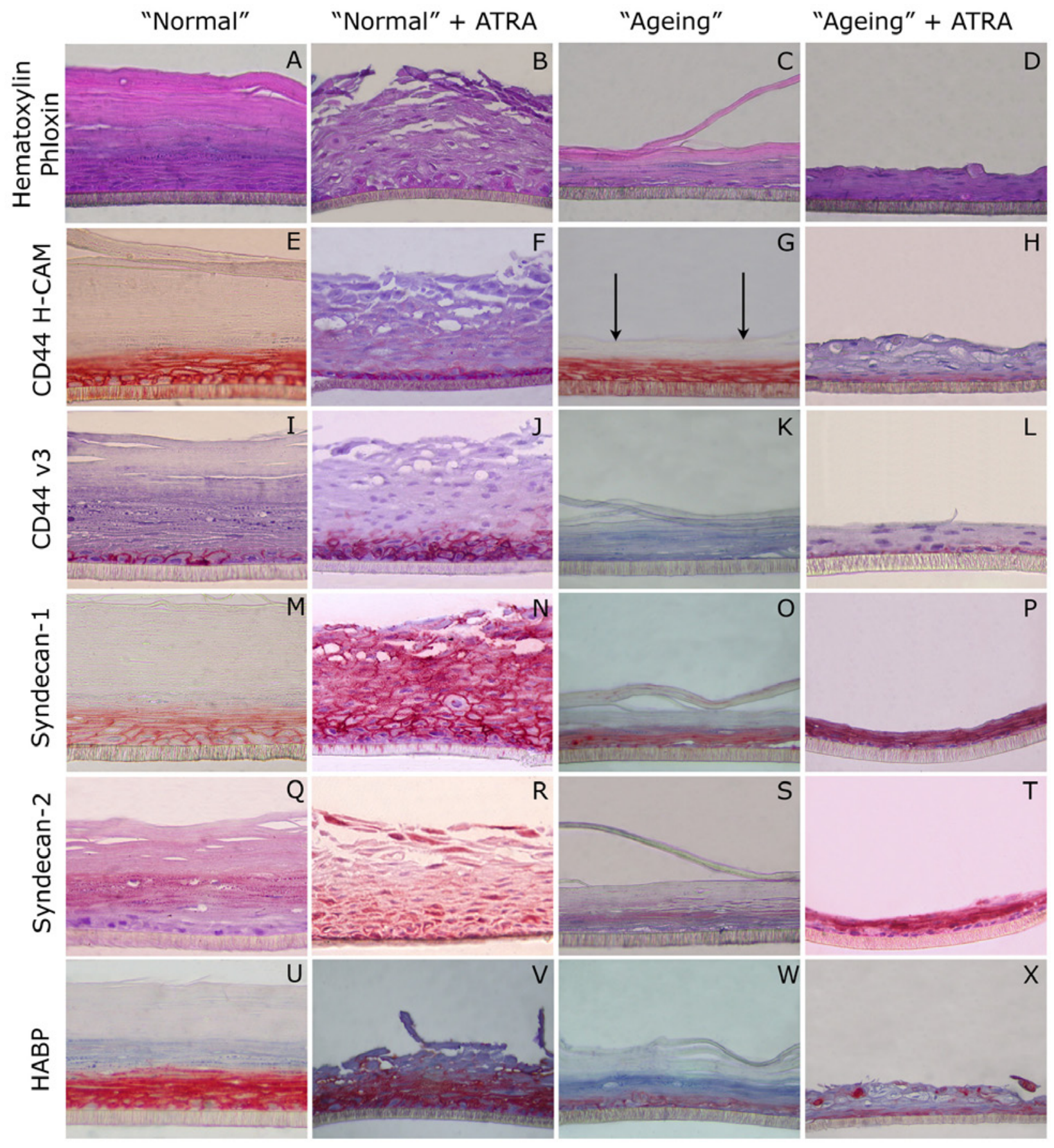

Fig. (2). Proteoglycan expression in reconstructed human epidermis follows morphological changes resulting from the culture conditions. Keratinocyte cultures on porous membranes at 14 days after lifting to the air-liquid interface. The reconstructed epidermis with thick retained stratum corneum was obtained with "normal" culture medium (first column). In the presence of a poorer "ageing" medium, thickness of the nucleated cell layers was markedly reduced, with the horny layer still representing approximately one-half of the total thickness (third column). Addition of all-trans retinoic acid ( $10 \mathrm{nM}$, during 8 final days of culture) resulted in a considerable increase in thickness of the nucleated viable compartment and in shedding of the cornified cells (second and fourth column), which was especially evident with the fully complemented "normal" medium (note disappearance of the pink horny layer from the cultures treated with ATRA, in the specimens routinely stained with hematoxylin-phloxine, A-D). E-H: CD44 H-CAM; I-L: CD44v3; M-P: Syndecan-1; Q-T: Syndecan-2; U-X: HABP, (no labeling was observed with the antibody to Syndecan-4). Arrows in G point to the surface of the stratum corneum. All "normal" and "ageing"-like cultures were Immunohistowax-embedded and labeled without previous antigen retrieval. All pictures were taken at x 400 magnification.

pressed biochemical and ultrastructural markers of epidermal differentiation, e.g., involucrin, filaggrin, corneodesmosin, keratohyalin granules, and lamellar bodies (data not shown).
The basal layer keratinocytes were attached to the polycarbonate membrane substrate through numerous minute extensions penetrating into the pores. 


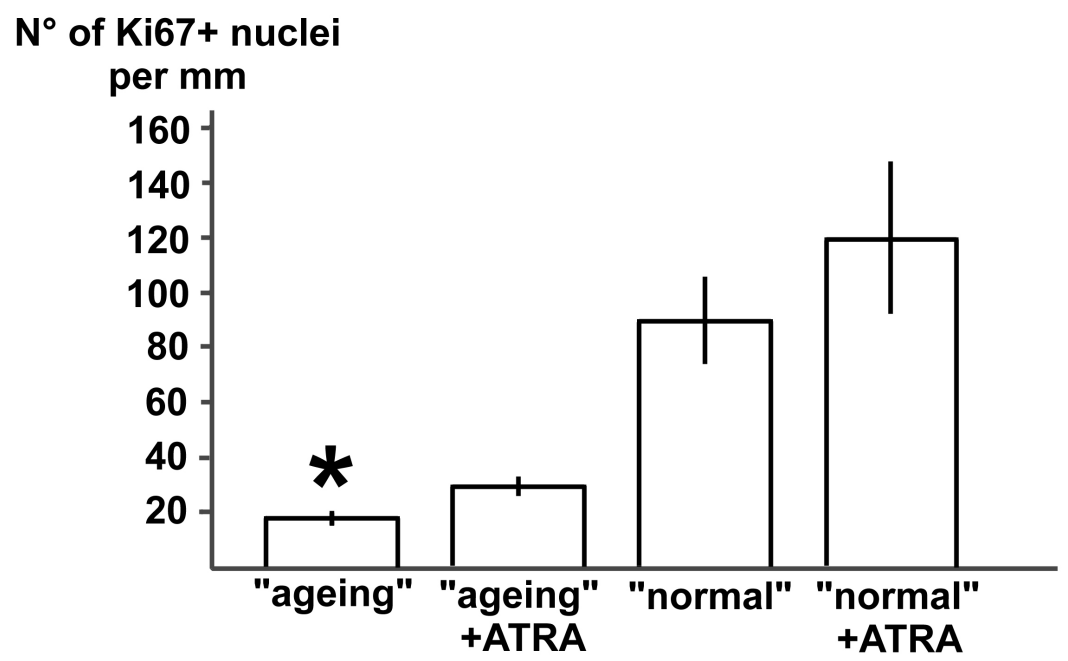

Fig. (3). Few Ki67 -positive cycling cells are observed in the "ageing"-like epidermis at 14 days after emersion (asterisk: P<0.01 when compared to the cultures maintained in the "normal" medium). Stimulation of the keratinocyte proliferation was achieved when 10 nM ATRA was added to one or another medium for the final 8 days. Representative photographs of Ki67 -labeled nuclei are inserted under each histogram indicating, for a given condition, mean number of Ki67+ cells per $1 \mathrm{~mm} \pm \mathrm{SD}$.

\section{“Ageing”-Like Reconstructed Epidermis Presented Prema- ture Differentiation which Could Be Partially Prevented by Treatment with all-Trans Retinoic Acid}

In the presence of the modified, poorer culture medium, our epidermal equivalent showed an accelerated formation of the horny layer combined with a marked reduction of the number of viable keratinocyte layers (Fig. 2, third column). Mean thickness of the viable epidermis in these "ageing"like cultures was $30.8 \mu \mathrm{m} \pm 5.5$ compared to $66.7 \mu \mathrm{m} \pm 5.5$ in cultures established under "normal" conditions (mean \pm $\mathrm{SD} ; P<0.01)$. Keratinocyte proliferation was also significantly reduced in these "ageing"-like cultures, as evidenced with Ki67 staining (Fig. 3). The late differentiation markers were always present.

After 8 days of ATRA treatment, the thickness of the viable nucleated epidermal layers in "ageing"-like cultures increased and the hyperkeratotic stratum corneum was shed (Fig. 2, fourth column). These changes were also observed in ATRA-treated "normal" cultures (Fig. 2, second column) but the increase of the viable portion was even more pronounced under these latter conditions.

\section{Immunohistochemical Detection of PGs in Various Cul- ture Systems}

All the antibodies directed to the epidermal PGs used in this study were able to detect the corresponding antigens in normal skin biopsies. Generally, the paraffin embedded tissues required antigen retrieval using microwave pretreatment. There was no notable difference observed between the expressions of various PGs according to the origin of the skin biopsy. Abdomen skin of adults (Fig. 4) showed the same pattern of staining as child foreskin. Also, cultured keratinocytes issued from both localizations gave similar staining results.

\section{CD44}

The hyaluronan receptor $\mathrm{CD} 44 \mathrm{H}-\mathrm{CAM}$ was strongly expressed in all epidermal tissues studied (Fig. 1A; Fig. 2E-
H; Fig. 4A). Its pericellular pattern of staining was observed from the basal layer on and persisted until the most superficial keratinocytes of the stratum spinosum. This distribution closely followed the presence of hyaluronic acid detected in the epidermal intercellular spaces with bHABP (Fig. 2U-X; Fig. 4C). Visible differences in the number of cell layers expressing CD44 were related mostly to the tissue architecture and thickness of the stratum spinosum, both largely depending on the conditions of cell culture. CD44 isoform recognized by the anti-CD44v3 antibody showed epidermal localization similar to H-CAM in normal human skin, but it was not detected on dermal cells (Fig. 4B). However, in the emerged keratinocyte cultures, its distribution was more restricted to the deepest epithelial layers, almost invisible in the "ageing"-like reconstructed epidermis, where only infrequently labeled basal cells could be observed (Fig. 2I,K). Treatment with ATRA resulted in an increase of the thickness of the stratum spinosum and a proportional spreading of the zone reactive with anti-CD44v3 MAb (Fig. 2J, L).

There was no influence of the preparative techniques used on the quality or extension of the CD44 staining, and equally so with both antibodies employed in this study.

\section{Syndecan-1}

The polyclonal anti-syndecan-1 antibody detected the extracellular fragment of the protein in all studied tissues, independent of the preparative approach (Fig. 1B; Fig. 2MP; Fig. 4D).

This PG was detected in the normal and cultured epidermis of all kinds. In all the cases, the pericellular staining of keratinocytes increased gradually from the basal to the lower granular layer. The upper granular keratinocytes showed considerably less labeling, especially in the cultures expressing a thick granular layer (Fig. 2M). After ATRA treatment, the cultured epidermis expressed syndecan-1 on its entire thickness. The granular layer was often disassembled on the sections of ATRA-treated samples, and there was no stratum corneum attached at the top of the epidermis (Fig. 2N, P). 


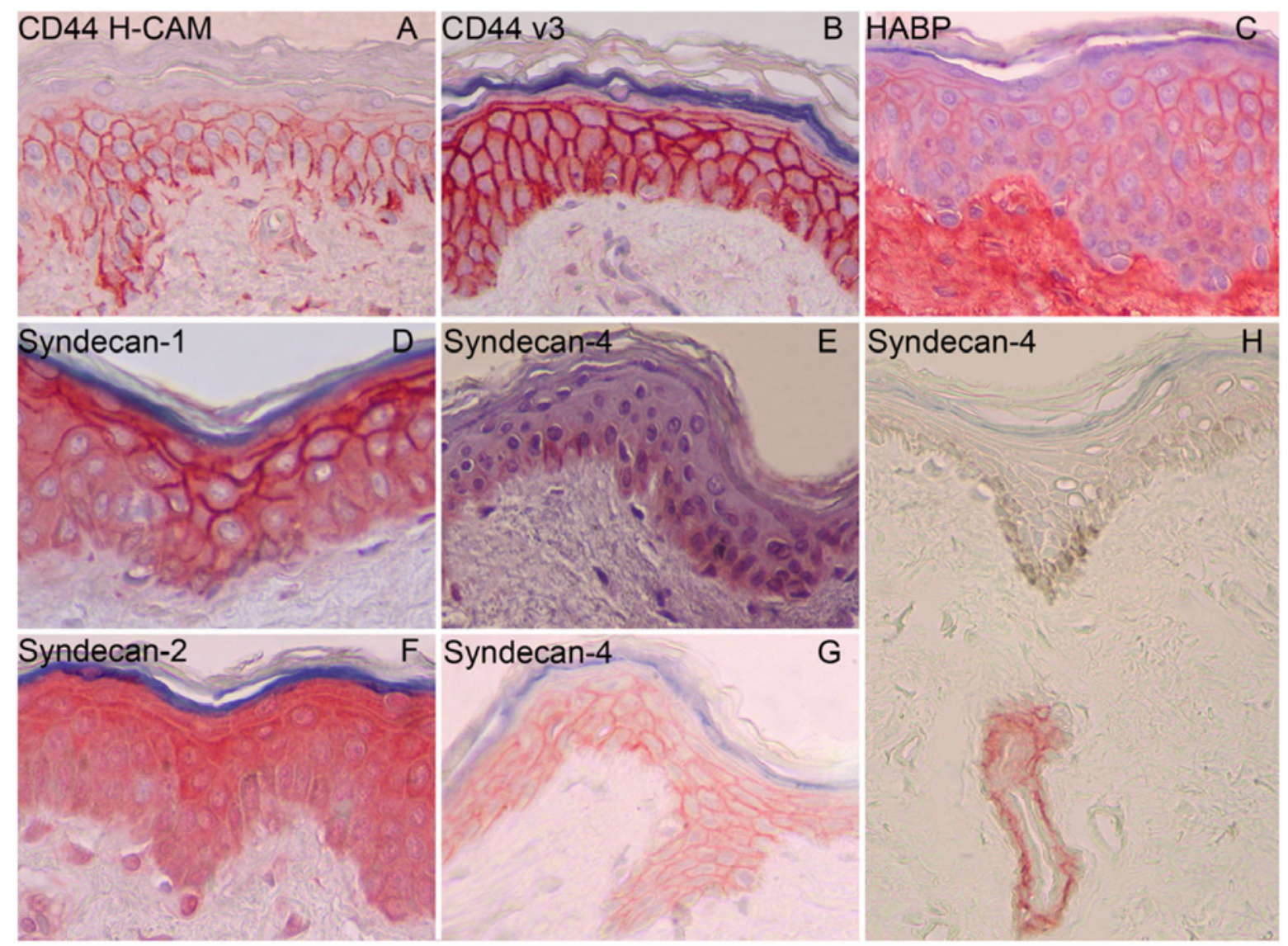

Fig. (4). Distribution of the studied epidermal proteoglycans in normal human skin (abdomen, adult). CD44 H-CAM (A), CD44v3 (B), syndecan-1 (D), and syndecan-4 (E, G, H) were distributed at the periphery of keratinocytes, whereas the antibody to syndecan-2 (F) gave a cytoplasmic staining. The antibody to H-CAM detected all forms of CD44, including these expressed on the dermal cells. Binding of bHABP, visualizing hyaluronan (C) was the strongest in the papillary dermis and its inter-keratinocyte distribution followed approximately the pattern of expression of CD44, the hyaluronan receptor. The staining of syndecans -1 and -2 was clearly the strongest in the better differentiated upper living layers ( $\mathbf{D}$ and $\mathbf{F}$, respectively). Reactivity of epidermal keratinocytes with the antibody to syndecan-4 was quite changeable: restricted to the lower epidermis on paraffin sections $(\mathbf{E}$; antigen retrieval) or distributed in all living layers on Immunohistowaxpreserved tissue $(\mathbf{G})$. Strong pericellular syndecan-4 expression by the eccrine gland epithelial cells was invariably present in all biopsies, even those presenting only traces of the epidermal staining (H; Immunohistowax) and thus constituted an internal positive standard. A-F: standard paraffin-embedded skin; G, H: Immunohistowax embedding. All pictures were taken at x 400 magnification.

\section{Syndecan-2}

The polyclonal antibody to the extracellular portion of syndecan-2 showed a cytoplasmic staining pattern in epidermal keratinocytes with all the preparative techniques employed (Fig. 1C; Fig. 2Q-T; Fig. 4F). Syndecan-2 labeling was predominantly situated in the upper layers of the viable part of the epidermis. Such a differentiation -related expression pattern was also observed in hair follicles (not shown) and was even more pronounced in the emerged keratinocyte cultures. The staining was diffuse in the upper stratum spinosum and in the granular layer, but only its traces could be observed within the fully keratinized cells. The emerged cultures maintained in the pro-ageing conditions showed a precocious keratinization and a syndecan-2 staining proportionally limited to a few mid-epidermal keratinocytes. The treatment with ATRA resulted in a moderate acanthosis and a delayed keratinization, introducing a stronger syndecan-2 expression, often beginning already in the basal layer (Fig. 2R, T).

\section{Syndecan-4}

The monoclonal anti-syndecan-4 antibody gave a pericellular staining pattern in the epithelial cells constituting eccrine sweat glands, whatever the substrate and the preparative techniques used (Fig. 3H). The presence of the staining in this dermal localization thus constituted our internal control for the antibody reactivity level. The epidermal staining was less reproducible and largely depended on the tissue preparation. ImmunoHistowax -embedded tissues reacted the best showing a pericellular keratinocyte staining situated in most cases mainly in the lower part of the epidermis (Fig. 3G). However, the staining never reached the granular layer and some biopsies remained totally non-reactive within the epidermal compartment (Fig. 3H). The cryosections of normal skin were stained within the basal layer only (not shown), whereas microwave antigen retrieval of dewaxed skin sections resulted in a variable degree of immunoreactivity of the basal layer keratinocytes (Fig. 3E). Reduced microwave energy ( 1 x 2 min. at $750 \mathrm{~W})$ had to be employed in 
Table 2. Amplification of the Proteoglycan Transcripts in Quantitative RT-PCR Assays Indicates a Retinoid-Induced Modulation of the Genes' Expression, Both in the "Normal" and "Ageing"-Like Culture Conditions

\begin{tabular}{|c|c|c|}
\hline Target & $\begin{array}{c}\text { Epidermal Cultures at “Normal” Conditions + ATRA (n=8) } \\
\text { Mean Change vs Untreated Cultures } \pm \text { SD }\end{array}$ & $\begin{array}{c}\text { Epidermal Cultures at “Ageing” Conditions + ATRA (n=8) } \\
\text { Mean Change } v \text { Untreated Cultures } \pm \text { SD }\end{array}$ \\
\hline \hline CD44 & $1.060 \pm 0.305$ & $1.137 \pm 0.132 *$ \\
\hline Syndecan-1 & $0.657 \pm 0.201 * *$ & $1.427 \pm 0.710$ \\
\hline Syndecan-2 & $1.300 \pm 0.366$ & $1.299 \pm 0.298 *$ \\
\hline Syndecan-4 & $1.685 \pm 0.326 * *$ & $1.484 \pm 0.355 * *$ \\
\hline
\end{tabular}

The values (arbitrary units) correspond to the variations of transcript quantities after treatment when compared to the untreated controls of the same kind (see materials and methods) All samples were first normalized against GAPDH. All-trans retinoic acid (ATRA, $10 \mathrm{nM}$ ) was applied to the emerged keratinocyte cultures established in either "normal" or "ageing" conditions for $24 \mathrm{~h}$. The controls received DMSO solvent only. Statistically significant differences (Wilcoxon T test): * p < 0.05; ** p < 0.01. SD: standard deviation.

order to avoid diffuse nuclear staining arising in sections treated following the ordinary protocol. Cultured epidermal keratinocytes were generally unable to produce syndecan-4 in amounts detectable with the antibody. It was even the case of emerged epidermal equivalents obtained in "normal" culture conditions, whether upon stimulation with ATRA or not.

\section{Measurement of PG mRNA Expression After ATRA Treatment Using Quantitative RT-PCR}

mRNAs coding for the four PGs were present both in normal human skin samples and in our "normal" and "ageing"-like emerged keratinocyte cultures, whether treated or not with ATRA. In normal skin samples, CD44 mRNA was the most abundant, followed by syndecan-1, syndecan- 2 and syndecan-4 (data not shown). The relative abundance of the different transcripts was inferred from the number of qRTPCR cycles necessary for the signal detection.

After treatment with ATRA, significant variations could be observed in the PG gene expression pattern (Table 2).

Expression of all PG mRNAs, Except Syndecan-1, was Increased by ATRA in "Ageing"-Like Emerged Keratinocyte Cultures

The level of syndecan-4 mRNA expression was found to be very significantly increased after 24 hour ATRA treatment in the "ageing"-like culture model. A significant upregulation of the CD44 and syndecan-2 genes was also observed, however that of syndecan-1 did not reach statistical significance.

In "Normal" Emerged Keratinocyte Cultures, ATRA Induced Syndecan-4 mRNA Expression and DownRegulation of the Syndecan-1 Gene

The levels of mRNA coding for syndecan-1 were found to be systematically decreased by approximately 40 percent when "normal" cultures were treated with ATRA. The syndecan-4 mRNA values increased significantly under the same conditions, as it was the case in the "ageing"-like epidermal cultures exposed to ATRA. No effect of ATRA was observed concerning CD44 mRNA expression and the increase of syndecan-2 gene expression was found to be insignificant in the "normal" cultures after $24 \mathrm{~h}$ of ATRA stimulation.

\section{DISCUSSION}

Despite their potentially fundamental role in the regulation of tissue biology, epidermal PGs remain poorly investi- gated. In an attempt to improve our knowledge about PG implication in the process of epidermal differentiation, we have created in vitro epidermal models mimicking normal and ageing-like conditions and modulated them pharmacologically. As expected, PGs known to be expressed in normal human epidermis could also be detected in the in vitro reconstructed tissues [31]. Although we could not localize the syndecan-4 protein immunohistochemically in any of the reconstructed epidermis used in this study, its mRNA was invariably detected in our culture system and, what is more, was significantly up-regulated by ATRA. The role of syndecan-4 remains unclear but it is believed to participate in epidermal wound healing. Mice with knocked out syndecan4 gene show delayed wound epithelialization [32] and the protein expression in keratinocytes that migrate across a fibrin clot is weaker compared with the hyperproliferative keratinocytes distal from the wound edge [25]. These observations are in agreement with our results showing an upregulation of syndecan- 4 gene in the retinoic acid - induced epithelial hyperplasia. The apparent absence of reactivity of epidermal keratinocytes with the antibody to syndecan-4 used in this study wasn't dependent on technical problems related to tissue fixation and/or embedding methods, since the epithelial structures of sweat glands were correctly detected on the normal skin sections. We propose that the differences may be rather due to the relative quantities of the expressed antigen.

David et al. found no syndecan-2 mRNA in mouse epidermis using in situ hybridization [33], whereas Kim et al. observed no syndecan-2 mRNA expression whatsoever in mouse epidermis by northern blot [9]. Gallo et al. also failed to demonstrate the syndecan-2 protein expression in tissue sections of human neonatal epidermis grafted on nude mice, however, they could detect it on a western blot [25]. Despite these largely unsuccessful initial approaches, syndecan-2 was finally localized in the viable epidermal layers by Lundqvist et al. [20]. We can additionally state that basal keratinocytes are rarely stained with the antibody to syndecan-2, unless the epidermis is treated with retinoids. ATRA induced up-regulation of the syndecan-2 gene was clearly demonstrated in the "ageing"-like reconstructed epidermis and preceded extension of the protein detection to the basal compartment. Just like the other molecules belonging to this family, syndecan- 2 is predicted to have transmembrane localization. Its exclusively cytoplasmic expression, confirmed ultrastructurally using the same antibody (data not shown), remains quite unusual and unexplained. 
Mice lacking syndecan-1 are viable, fertile and have morphologically normal skin, hair and ocular surface epithelia. Nevertheless, in these mice, keratinocytes failed to activate cell proliferation in response to wounding, resulting in delayed wound healing [26]. On the other hand, proliferation of keratinocytes at wound edges was equally delayed in mice over-expressing syndecan-1 [27]. We have shown in this study that, depending on the initial condition of a treated culture, syndecan- 1 gene expression was differentially regulated by retinoic acid. When keratinocytes were grown in the poor culture medium inducing "ageing"-like changes, the addition of retinoic acid did not provoke a significant change in syndecan-1 gene transcription. In contrast, when the fully supplemented "normal" medium was used, cultured keratinocytes reduced significantly syndecan-1 mRNA production. These observations are in concordance with the recognized "regulatory" action of the retinoids, known to influence both keratinocyte proliferation and differentiation, often one at the expense of the other.

Taken together, our results support the notion of a fine regulation of syndecan-1 during keratinocyte differentiation. Specifically, an "invasion regulatory domain" has been detected on this PG's ectodomain by Langford et al. [34]. Its apparent involvement in the inhibition of tumor cell invasion may explain the observed relationship between the decrease or loss of syndecan-1 and highly invasive behavior of some cancer cells [35-37].

The CD44 primer couple used in our study was designed to amplify the mRNAs of all the CD44 variants. The increase of the generic CD44 signal was consistently observed in the "ageing"-like keratinocyte cultures but not in the "normal" conditions, suggesting that the anti-ageing action of ATRA was associated with the up-regulation of CD44.

Indeed, an up-regulation of all CD44s by retinaldehyde topically applied on mouse skin (immunohistochemical detection and northern blot) has been observed by Kaya et al. and linked by these authors to the co-receptor function of this PG in heparin binding EGF receptor -mediated growth stimulation [38]. However, a moderate decrease of the total human CD44 gene transcripts has been described using a cDNA array approach when retinoids were applied in the human keratinocyte culture media, in the SkinEthic model [39]. It is likely that specific CD44 species, like the functionally important v3 variant, are regulated independently and differently from the other alternatively spliced isoforms. Indeed, some differences in the expression of CD44 variants during keratinocyte proliferation and differentiation were previously described by Zhou et al., with a general downregulation of CD44 expression in the differentiated cells compared to the proliferating ones [15]. Moreover, the relative molecular weight of the variant $\mathrm{v} 3$ expressed on differentiated keratinocytes was smaller than that found on basal cells, suggesting an important variation in the glycosylation of the protein. It would therefore be interesting to refine our analysis using the primer couples specific for each CD44 variant, in order to better understand the regulation of this proteoglycan family.

In view of a large variability of the CD44 response observed in our highly normalized emerged keratinocyte cultures, we insist on the necessity of several qRT-PCR assays to be performed before the reliable quantitative results may be proposed. Indeed, despite the use of a keratinocyte mix originating from three carefully chosen donors, designed to level the differences between the studied cultures, we could not completely avoid the problem of such inter-experiment variability, which could only be overcome by multiplication of the assays.

Although promising, the qRT-PCR studies have to be performed in concert with the protein expression assays since some PG expression can also be controlled posttranscriptionally. For instance, syndecan-1 expression has been shown to increase 3-fold, without a change in mRNA levels, in stratifying keratinocytes when compared to the cells in a monolayer [23]. Inversely, the presence of mRNA coding for a given PG does not necessarily mean the presence of the protein, and even less the expression of a fully glycosylated and functionally modified form of the PG.

Our reconstructed epidermis represented two differentiation states, depending on the culture conditions: "normal" and "ageing"-like. Natural senescence of keratinocytes is most probably related to the decrease of the metabolic activity of the cells. Such a slowing down of keratinocyte metabolism can be achieved by modulation of the culture conditions, leading to the same morphological outcome characteristic of the "aged" epidermis. We used this approach to reproduce the morphologic criteria of "epidermal ageing" in vitro. The validation of this model was accomplished using molecular, histological, and ultrastructural methods of analysis combined with the pharmacological modulation assays employing a reference molecule, the all-trans retinoic acid. Our results underline the relationship between the PG expression and regulation of keratinocyte differentiation.

\section{CONCLUSIONS}

This study demonstrates the utility of a human epidermal equivalent cultured in fully defined conditions for the screening of pharmacological compounds, in search of their effects on epidermal PG expression.

Epidermal differentiation is a finely regulated process involving the sequential expression of various structural molecules and epidermal PG are no exception to this rule. Our results support, specifically, the notion of a precise regulation of syndecan-1 during keratinocyte differentiation. Because glycosaminoglycan chains expression can largely modulate functional properties of $\mathrm{PG}$, further studies on glycosylation are required to better understand the role(s) of these complex and biologically important molecules during epithelial differentiation, wound healing, and senescence.

\section{ACKNOWLEDGEMENTS}

We thank the "Analytical Cytology Laboratory" and the "Medical Parasitology - Mycology Laboratory" at the University of Lyon 1 for granting us access to their qPCR equipment.

We are grateful to Dr. Anne Beghin for her valuable technical suggestions and helpful discussions and to Dr. Monique Arlot for help in the statistical analysis. M.-A. Le Bitoux was a PhD student, a holder of CIFRE fellowship, supported by the Bioderma Laboratory and by the French National Association of Technical Research (ANRT). 


\section{ABBREVIATIONS}

$$
\begin{array}{ll}
\text { ATRA } & =\text { All-trans retinoic acid } \\
\mathrm{bHABP} & =\text { Biotinylated hyaluronic acid binding protein } \\
\mathrm{MAb} & =\text { Monoclonal antibody } \\
\mathrm{PG} & \text { Proteoglycan } \\
\mathrm{qRT}-\mathrm{PCR}= & \text { Quantitative real-time polymerase chain } \\
& \text { reaction }
\end{array}
$$

\section{REFERENCES}

[1] Hardingham TE, Fosang AJ. Proteoglycans: many forms and many functions. FASEB J 1992; 6: 861-70.

[2] Iozzo RV. Matrix proteoglycans: from molecular design to cellular function. Annu Rev Biochem 1998; 67: 609-52.

[3] Knudson C, Knudson W. Hyaluronan-binding proteins in development, tissue homeostasis, and disease. FASEB J 1993; 7: 1233-41.

[4] Bernfield M, Kokenyesi R, Kato M, et al. Biology of the syndecans: a family of transmembrane heparan sulfate proteoglycans. Annu Rev Cell Biol 1992; 8: 365-93.

[5] Kramer KL, Yost HJ. Heparan sulfate core proteins in cell-cell signaling. Annu Rev Genet 2003; 37: 461-84.

[6] Carey DJ. Syndecans: multifunctional cell-surface co-receptors. Biochem J 1997; 327: 1-16.

[7] Bernfield M, Gotte M, Park PW, et al. Functions of cell surface heparan sulfate proteoglycans. Annu Rev Biochem 1999; 68: 729-77.

[8] Fitzgerald ML, Wang Z, Park PW, Murphy G, Bernfield M. Shedding of syndecan-1 and -4 ectodomains is regulated by multiple signaling pathways and mediated by a TIMP-3-sensitive metalloproteinase. J Cell Biol 2000; 148: 811-24.

[9] Kim CW, Goldberger OA, Gallo RL, Bernfield M. Members of the syndecan family of heparan sulfate proteoglycans are expressed in distinct cell-, tissue-, and development-specific patterns. Mol Biol Cell 1994; 5: 797-805.

[10] Fears CY, Gladson CL, Woods A. Syndecan-2 is expressed in the microvasculature of gliomas and regulates angiogenic processes in microvascular endothelial cells. J Biol Chem 2006; 281: 14533-6.

[11] Kainulainen V, Wang H, Schick C, Bernfield M. Syndecans, heparan sulfate proteoglycans, maintain the proteolytic balance of acute wound fluids. J Biol Chem 1998; 273: 11563-9.

[12] Kato M, Wang H, Kainulainen V, et al. Physiological degradation converts the soluble syndecan-1 ectodomain from an inhibitor to a potent activator of FGF-2. Nat Med 1998; 4: 691-7.

[13] Aruffo A, Stamenkovic I, Melnick M, Underhill CB, Seed B. CD44 is the principal cell surface receptor for hyaluronate. Cell 1990; 61: 1303-13.

[14] Bloor BK, Rajarajan A, Jaafary-Haghighat K, Odell EW. Transcription and expression of CD44 variant exons by oro-pharyngeal squamous cell carcinomas. Int J Oncol 2002; 21: 907-13.

[15] Zhou J, Haggerty JG, Milstone LM. Growth and differentiation regulate CD44 expression on human keratinocytes. In Vitro Cell Dev Biol Anim 1999; 35: 228-35.

[16] Ponta H, Sherman L, Herrlich PA. CD44: from adhesion molecules to signalling regulators. Nat Rev Mol Cell Biol 2003; 4: 33-45.

[17] Milstone LM, Hough-Monroe L, Kugelman LC, Bender JR, Haggerty JG. Epican, a heparan/chondroitin sulfate proteoglycan form of CD44, mediates cell-cell adhesion. J Cell Sci 1994; 107: 3183-90.

[18] Kugelman LC, Ganguly S, Haggerty JG, Weissman SM, Milstone LM. The core protein of epican, a heparan sulfate proteoglycan on keratinocytes, is an alternative form of CD44. J Invest Dermatol 1992; 99: 381-85.

[19] Haggerty JG, Bretton RH, Milstone LM. Identification and characterization of a cell surface proteoglycan on keratinocytes. J Invest Dermatol 1992; 99: 374-80.
[20] Lundqvist K, Schmidtchen, A. Immunohistochemical studies on proteoglycan expression in normal skin and chronic ulcers. Br J Dermatol 2001; 144: 254-9.

[21] Calikoglu E, Sorg O, Tran C, et al. UVA and UVB decrease the expression of CD44 and hyaluronate in mouse epidermis, which is counteracted by topical retinoids. Photochem Photobiol 2006; 82: 1342-7.

[22] Kaya G, Rodriguez I, Jorcano JL, Vassalli P, Stamenkovic I. Selective suppression of CD44 in keratinocytes of mice bearing an antisense CD44 transgene driven by a tissue-specific promoter disrupts hyaluronate metabolism in the skin and impairs keratinocyte proliferation. Genes Dev 1997; 11: 996-1007.

[23] Sanderson RD, Hinkes MT, Bernfield M. Syndecan-1, a cell-surface proteoglycan, changes in size and abundance when keratinocytes stratify. J Invest Dermatol 1992; 99: 390-6.

[24] Elenius K, Vainio S, Laato M, Salmivirta M, Thesleff I, Jalkanen M. Induced expression of syndecan in healing wounds. J Cell Biol 1991; 114: 585-95.

[25] Gallo R, Kim C, Kokenyesi R, Adzick NS, Bernfield M. Syndecans-1 and -4 are induced during wound repair of neonatal but not fetal skin. J Invest Dermatol 1996; 107: 676-83.

[26] Stepp MA, Gibson HE, Gala PH, et al. Defects in keratinocyte activation during wound healing in the syndecan-1-deficient mouse. J Cell Sci 2002; 115: 4517-31.

[27] Elenius V, Gotte M, Reizes O, Elenius K, Bernfield M. Inhibition by the soluble syndecan-1 ectodomains delays wound repair in mice overexpressing syndecan-1. J Biol Chem 2004; 279: 41928-35.

[28] Yaar M, Eller MS, Gilchrest BA. Fifty years of skin aging. J Invest Dermatol Symp Proc 2002; 7: 51-8.

[29] Boyce ST, Williams ML. Lipid supplemented medium induces lamellar bodies and precursors of barrier lipids in cultured analogues of human skin. J Invest Dermatol 1993; 101: 180-4.

[30] Ponec M, Weerheim A, Kempenaar J, et al. The formation of competent barrier lipids in reconstructed human epidermis requires the presence of vitamin C. J Invest Dermatol 1997; 109: 348-55.

[31] Le Bitoux MA, Callejon S, Denis A, et al. In vitro reconstructed normal human epidermis expresses differentiation-related proteoglycans. J Dermatol Sci 2008; 51: 135-8.

[32] Echtermeyer F, Streit M, Wilcox-Adelman S, et al. Delayed wound repair and impaired angiogenesis in mice lacking syndecan-4. J Clin Invest 2001; 107: R9-R14.

[33] David G, Bai XM, Van der Schueren B, Marynen P, Cassiman JJ, Van den Berghe H. Spatial and temporal changes in the expression of fibroglycan (syndecan-2) during mouse embryonic development. Development 1993; 119: 841-54.

[34] Langford JK, Yang Y, Kieber-Emmons T, Sanderson RD. Identification of an invasion regulatory domain within the core protein of syndecan-1. J Biol Chem 2005; 280: 3467-73.

[35] Anttonen A, Kajanti M, Heikkila P, Jalkanen M, Joensuu H. Syndecan-1 expression has prognostic significance in head and neck carcinoma. Br J Cancer 1999; 79: 558-64.

[36] Anttonen A, Heikkila P, Kajanti M, Jalkanen M, Joensuu H. High syndecan-1 expression is associated with favourable outcome in squamous cell lung carcinoma treated with radical surgery. Lung Cancer 2001; 32: 297-305.

[37] Wiksten JP, Lundin J, Nordling S, et al. Epithelial and stromal syndecan-1 expression as predictor of outcome in patients with gastric cancer. Int J Cancer 2001; 95: 1-6.

[38] Kaya G, Grand D, Hotz R, et al. Upregulation of CD44 and hyaluronate synthases by topical retinoids in mouse skin. J Invest Dermatol 2005; 124: 284-7.

[39] Bernard FX, Pedretti N, Rosdy M, Deguercy A. Comparison of gene expression profiles in human keratinocyte mono-layer cultures, reconstituted epidermis and normal human skin; transcriptional effects of retinoid treatments in reconstituted human epidermis. Exp Dermatol 2002; 11: 59-74. 